\title{
Merging of "MnM" and BallTech Technologies to Create a Unique Manganese Removal Filtration System
}

\author{
Roy Elkayam ${ }^{1}$, Ilan Kessler ${ }^{2}$, Ovadia Lev ${ }^{3}$ \\ ${ }^{1}$ Mekorot Water Company and Hebrew University of Jerusalem \\ Lincoln St., 9, P.O.B. 20128, Tel Aviv, 6120101, Israel \\ relkayam@mekorot.co.il; \\ ${ }^{2}$ BallTech Energy Ltd \\ Ha'amal st., 24, Park Afeq Rosh Haaiyn \\ ilan.kessler@balltech.co.il \\ ${ }^{3}$ Institute of Chemistry, Hebrew University of Jerusalem \\ Jerusalem, 91904, Israel \\ ovadia@mail.huji.ac.il
}

\section{Extended Abstract}

A new manganese-based filter media named "MnM" has been developed at Mekorot, the National Water Company of Israel, for the removal of dissolved manganese from water. A patent application has been filed. The process that was developed uses a large quantity of anthracite supported MnM. and enables aerobic conditions within the filter itself. Analysis of the water which passes through the filter shows that the residual level of manganese after filtration is negligible. The new reactive filter does not require regeneration, nor the addition of chemicals. The MnM filter is a proven technology for manganese removal, based on adsorption of manganese from water as it passes through the filter media. At the same time, catalytic oxidation occurs on the filter bed itself, while simultaneously regenerating the MnM substance. The MnM filter is a unique sustainable process which is "chemical free".

BallTech Energy new concept (patent application has been filed) Whirled Sand Media Filter (WSMF) is a fully automatic no backwash filter specially designed for stick particles filtration by using very little sand rinsing water. The WSMF filter installed on a variety application like condenser and chilled cooling system water and industrial waste water filtration. Where every single sand grain in washed as a single unit. There is no filter clogging in the WSMF filter, no sand replacement and sand rinsing require very little water.

Merging these technologies provides elegant solution for small scale system that require manganese removal. The system appears to be compact and easy for operation in the field with less than $0.0001 \%$ water loss and no chemical addition. A pilot located at "Yavne" area, Israel was operating in a flow between 1-3 m3/h.

The pilot research on the merging of MnM \& Balltech filtration system shows that while the condition in the filter were aerobic, more than $90 \%$ of the manganese was removed from influent containing about $0.8 \mathrm{mg} / \mathrm{lof}$ dissolved manganese. The sand rinsing conditions were once every 10 days, the water from sand rinsing was about 12 liter, the sand rinsing water passed through Geotube filter that catches all the solids. The Geotube filtrated water seems to be clean enough to be inserted back to the filtration inlet stream, reducing even more the water loss. 